\title{
Community Care und Community Living
}

\author{
Kritische Anmerkungen zu einer Diskussion in der Behindertenhilfe
}

Jens Jürgen Clausen

\begin{abstract}
Gemeindeorientierung und Inklusion sind die neuen Leitbegriffe in der Behindertenbilfe. Die unkritische Übernahme dieser Vorstellungen birgt jedoch die Gefabr in sich, dass viele Menschen mit einer Behinderung auf eine nachbarschaftliche Hilfe verweisen werden, die es gar nicht gibt.
\end{abstract}

Der Umbau der sozialen Sicherungssysteme in Bezug auf Arbeitsmarktpolitik oder Gesundheitspolitik vollzieht sich spürbar und geräuschvoll - eine gut sichtbare Baustelle mit vielen Architekten, die allerdings nicht alle den gleichen Plan vor Augen zu haben scheinen. Die Öffentlichkeit verfolgt diesen Umbau mit Spannung, Enttäuschung oder Empörung, jedenfalls höchst wachsam und emotional. Dagegen vollziehen sich die Umbaumaßnahmen auf der Baustelle Behindertenhilfe eher lautlos und unterhalb der öffentlichen Wahrnehmung. Obwohl vor der verhüllten Fassade des alten Gebäudes jedes Jahr ein neues Plakat angebracht wird, auf dem dann »Community Care« oder »Community Living « oder »Inklusion « oder »Persönliches Budget « steht, geht das Publikum recht achtlos daran vorüber. Wie es in dem entkernten Objekt einmal aussehen soll, das haben nur wenige Insider deutlich vor Augen.

Aufseiten der Kostenträger und Leistungserbringer wird der Diskurs über die genannten Begriffe und Perspektiven hingegen mit großem Engagement geführt. Es geht um die Frage, welche Unterstützung die Gesellschaft für ihre behinderten Bürgerinnen und Bürger bereithält und (noch) zu finanzieren bereit ist. Und so werden seit einiger Zeit die Strukturen

Dr. Jens Jürgen Clausen lehrt an der Evangelisch Sozialpädagogischen Ausbildungsstätte in Münster und an der Westfälischen Wilhelm-

Universität Münster im Fachbereich Erziehungswissenschaft.

E-Mail jjclausen@web.de und Institutionen der Versorgung und Pflege behinderter Menschen von der Politik und der Sozialverwaltung auf ihre Zukunftsfähigkeit überprüft. Dieser Prozess hat in den letzten Monaten deutlich an Tempo und Intensität zugenommen. Die Gründe dafür liegen - aus Sicht der Kostenträger - auf der Hand:

Die größte Gruppe der Leistungsempfänger der Eingliederungshilfe sind Menschen mit Behinderungen im Alter von 40 bis 55 Jahren. Diese Gruppe wächst ins Alter hinein, ihre Pflegebedürftigkeit nimmt zu. Damit werden, wenn nicht in absehbarer Zeit eine gewisse Umsteuerung geschieht, die Kosten der Eingliederungshilfe erheblich steigen. Dazu haben aber weder die örtlichen noch die überörtlichen Sozialhilfeträger die notwendigen finanziellen Ressourcen - und die Pflegekassen auch nicht. Daher weisen Gesetzgeber und Kostenträger jetzt verstärkt auf das Gebot »ambulant vor stationär « hin und meinen eigentlich »ambulant statt stationär«. Ihre Argumentation ist gefüllt mit Begriffen wie sozialräumlich, lebensweltlich, selbstbestimmt und eigenverantwortlich. Und an dieser Stelle tauchen dann auch die Konzepte von Community Care und Community Living auf.

Community meint: Gemeinschaft, Körperschaft, Gemeinde, Gemeinwesen. Care bedeutet: Sorge, Fürsorge, Aufmerksamkeit, ursprünglich auch: Besorgnis, Aufsicht, Obhut, Pflege. Community Care soll also heißen: Die Gemeinschaft, die Gemeinde, das Gemeinwesen übernimmt die Aufmerksamkeit für ihre Bürgerinnen und Bürger, auch und gerade für jene, die behindert sind - oder behindert werden. Ein Gemeinwesen wird aber erst dann zu einer Gemeinde und zu einer Gemeinschaft, wenn sie nicht nur über ein adäquates Angebot an Beratung und Unterstützung verfügt, sondern wenn auch die in ihr lebenden Bürgerinnen und Bürger Sorge tragen für Menschen mit Behinderungen, wie auch für alle anderen Men- schen, die der Unterstützung oder Pflege bedürfen. Wenn das gelänge, dann könnte Community Care, so sagen ihre Fürsprecher, eine Vertiefung des Miteinanders in der Gesellschaft eröffnen und das Leben aller reicher machen.

Dieser Aspekt wird auch von den Protagonisten des Community Living betont: Damit Menschen mit Behinderung ihre volle Teilhabe an der Gesellschaft wahrnehmen können, so die Europäische Koalition für Community Living in Brüssel, brauchen sie Zugang zu umfassenden Dienstleistungen. Doch diese sollen ihren Sitz nicht abseits, sondern mitten in der Gemeinde haben. Menschen mit Behinderungen sollen unabhängig leben können, in kleinen Wohneinheiten oder allein, mit passgenauer Unterstützung, die sich nach den Bedürfnissen des Einzelnen richtet. Und sie sollen Zugang haben zu Bildung, Beschäftigung und zum sozialen Leben in der Gemeinde.

Ausgangspunkt von Community Living ist die Überzeugung, dass Menschen mit Behinderung selbst bestimmen können und wollen, wie sie ihrem Leben Form und Inhalt geben. Während Community-Care dabei stärker die Form der Unterstützung prüft, hebt CommunityLiving den gemeinwesenorientierten Charakter des Prozesses hervor. In diesem Zusammenhang taucht auch der Begriff der Inklusion verstärkt auf. Inklusion bedeutet wörtlich: Einschluss, Einbeziehung und fordert dazu auf, Menschen mit Behinderungen das notwendige Maß an Unterstützung anzubieten, ihnen einen Zugang zu gesellschaftlichen Regelkontakten zu ermöglichen, eine Teilhabe an gesellschaftlichen Bezügen zu gewährleisten und ihnen ein Leben in ihrer vertrauten Umgebung zu sichern.

So ist der Begriff Inklusion eng verknüpft mit den Konzepten Community Care und Community Living und tritt die Nachfolge der Prinzipien der Normalisierung und Integration an und weist über sie hinaus. Nicht mehr die bloße Einglie- 
derung behinderter Menschen in die Gesellschaft wird angestrebt, sondern die Umgestaltung der Community im Sinne einer inklusiven Gesellschaft. Knapp formuliert: »Integration bedeutet Ausgrenzung aufheben, Inklusion dagegen, Ausgrenzung von vornherein gar nicht erst zuzulassen. «(Frühauf/Niehoff 2007)

An dieser Stelle ist zu fragen, ob die Behindertenhilfe gut beraten ist, den Grad der Komplexität und Differenziertheit unserer modernen Gesellschaft gänzlich unberücksichtigt zu lassen und Begriffe wie Gemeinde und Gemeinschaft romantisch zu überhöhen. Muss man nicht heute - vor dem Hintergrund tiefgreifender sozialer Umbrüche - feststellen, dass Prozesse des Ausschlusses, der Exklusion aus wichtigen gesellschaftlichen Bezügen keineswegs beschränkt sind auf Menschen mit Behinderungen, ja noch nicht einmal auf wenige marginalisierte Personengruppen? Ist es zulässig, den Begriff der Inklusion aus seiner wissenschaftstheoretischen Verankerung zu lösen und unkritisch im Sinne einer Formulierung von Solidaritätserwartungen zu verwenden?

Meist wird in der aktuellen Debatte Inklusion zu einer normativen Leitidee stilisiert und das Konzept, welches man damit verbindet, lediglich in den Kontext von Vergangenheit und Gegenwart gestellt: Bislang wurden Menschen mit Behinderungen ausgegrenzt, nun soll ihre volle Teilhabe am gesellschaftlichen Leben selbstverständlich sein - oder, um es in Abwandlung von Freuds Diktum vom Es und vom Ich zu formulieren: » Wo Exklusion war, soll Inklusion werden. « Eine solche Vereinfachung des Inklusionsbegriffs ist, wie gesagt, in wissenschaftstheoretischer Hinsicht erschreckend und in sozialwissenschaftlicher Hinsicht kurzsichtig - auch und gerade im Sinne einer engagierten Parteinahme für Menschen, deren Leben bislang als randständig - in Bezug auf die "Mitte der Gesellschaft « angesehen wurde.

Dabei wissen wir schon lange, dass eine moderne, funktional differenzierte Gesellschaft nirgendwo einen für alle verbindlichen Ort hat, an dem ihre Kohärenz zum Ausdruck kommt: "Sie artikuliert ihre Einheit weder über eine Rangordnung der Schichten, noch über eine Herrschaftsordnung, noch über eine Lebensform.« (Luhmann 1995) Prozesse der Teilhabe und des Ausschlusses, der Inklusion wie der Exklusion, werden in diffe- renzierten Gesellschaften vielmehr durch die einzelnen Funktionssysteme und ihre Kommunikationszusammenhänge gesteuert. Die moderne Gesellschaft schließt eigentlich niemanden komplett in sich ein, im Gegenteil, sie produziert laufend Ein- und Ausschlüsse. Diese Seite der Medaille bleibt jedoch in der aktuellen Diskussion um neue Konzepte in der Behindertenhilfe unerwähnt.

Und noch ein weiterer Aspekt, der in den Konzepten von Community Care, Community Living und Inklusion eine Rolle spielt, ist zu erwähnen, nämlich die unkritische Übernahme des Begriffs Lebensweltorientierung. Neben der kommu-
Menschen mit geistiger Behinderung in den Blick zu nehmen und offensiv für geeignete Lebensräume und Lebensqualität einzutreten." (Theunissen 2007).

An solchen wohlmeinenden Aussagen wird deutlich: Manche Position der neuen Behindertenhilfe greift ganz unhinterfragt klassische Vorstellungen der Sozialen Arbeit auf, die im aktuellen Diskurs längst eine Vertiefung oder kritische Überprüfung erfahren haben. So weist eine systemtheoretisch orientierte Sozialarbeitswissenschaft heute zu Recht darauf hin, dass man besser zwischen Lebenslage und Lebenswelt differenzieren sollte. Und sie tut dies nicht aus Spitzfindigkeit, sondern

\section{" Community Care steht im Grunde für das intakte soziale Umfeld - das viele behinderte Menschen nicht haben"}

nalen Steuerung und der individuellen Hilfeplanung ist nun auch die Lebensweltorientierung in der Behindertenhilfe angekommen. Wenig beachtet wird dabei jedoch der Diskussionszusammenhang, in welchem dieser Begriff steht: Als in den 1980er Jahren das Konzept »Lebensweltorientierte Soziale Arbeit« Bedeutung erlangte, so geschah dies kontrapunktisch zur bis dahin sehr symptomorientierten Betrachtungsweise. Nicht individuelle Defizite und Förderaspekte sollten im Fokus stehen, sondern »der Mensch in seinen gegebenen Lebensverhältnissen, also in seiner Bezogenheit auf andere und seinem Eingebettet-Sein in die materiellen, sozialen und symbolischen Strukturen der Lebenswelt, in denen er sich immer schon vorfindet « (Thiersch 2005). Daraus leiten die Community-Living-Vertreter nun die Vorstellung ab, dass Maßnahmen zur Assistenz in der Behindertenhilfe ihre Bedeutung erst durch die Einbeziehung in die lebensweltlichen Zusammenhänge des betroffenen Menschen erhalten: "Dies bedeutet, dass bei allen Formen einer Unterstützung, Förderung, Therapie stets das soziale Bezugsfeld als autonomiehemmender oder -fördernder Faktor mitreflektiert und berücksichtigt werden muss. (...) Somit gehört es zu einem wichtigen (insbesondere auch präventiven) Anliegen der Behindertenhilfe, soziale Kontexte von aus der Erkenntnis heraus, dass man sich in der Praxis kaum an den »unhintergehbar subjektiven und deshalb nicht direkt zugänglichen Wirklichkeitskonstruktionen « (Kraus 2007) eines Menschen orientieren kann. Anders formuliert: Selbst wenn sich die Lebenslage eines Menschen gut ermittelt lässt und ein Eindruck davon entsteht, wie seine Verfügungsmöglichkeiten über materielle Ressourcen und seine Kontakte im sozialen Netzwerk beschaffen sind, so bedarf es doch einer behutsamen, empathischen Begleitung, um ein Gespür für subjektive Wahrnehmungen und Wirklichkeitskonstruktionen zu erlangen. Und selbst dann wird klar: Die Lebenswelt ein Menschen lässt sich nicht wirklich erfassen. Wir können lediglich Hypothesen über individuelle Wirklichkeitskonstruktionen eines Menschen aufstellen, unabhängig davon, ob er behindert ist oder nicht. Und wir können unsere Einfühlungsversuche schulen; was besonders wichtig ist, wenn die üblichen kommunikativen Wege nicht greifen.

Natürlich ist es notwendig, die Formen von Fremdbestimmung, Abhängigkeit und Entmündigung, die oft und anhaltend in der Betreuung behinderter Menschen anzutreffen waren, aufzudecken und zu verändern. Aber: Menschen mit Behinderungen wurden und werden nicht nur abhängig gemacht, sie sind auch ver- 
letzbar - und angewiesen auf kompetente Unterstützung. Das sehen Betroffene, die sich organisiert und in die Debatte um Community Care und Communty Living eingemischt haben, ganz ähnlich. So mahnt Gerlef Gleiss von der Gruppe »Selbstbestimmt Leben $«$ :

»Eine große Gefahr im Konzept der Communty Care liegt unter den gegenwärtigen sozialen und wirtschaftlichen Bedingungen darin, dass von dem wunderbaren Vorhaben nur übrigbleibt, dass sich der Staat aus seiner sozialpolitischen Verantwortung zurückzieht - und dass bisher sozialhilferechtlich gesicherte und geschützte Hilfen unter Verweis auf die jetzt dafür verantwortliche ,Community< abgeschafft werden. >Community Carer steht ja im Grunde für nichts anderes als für das schnöde ssoziale Umfeld : freundschaftliche Hilfe durch Bekannte, Angehörige oder Nachbarn. Diese Hilfe soll vorrangig genutzt werden, bevor professionelle Helfer hinzukommen. Das Bedrohliche daran ist nur, dass viele behinderte Menschen dieses intakte, zur Unterstützung bereitstehende soziale Umfeld nicht haben - und trotzdem bei der Feststellung ihrer Hilfebedarfe darauf verwiesen werden. Und noch schlimmer und ein nicht hinnehmbarer sozialpolitischer Rückschritt wäre es, wenn die behinderten Menschen auf diesem Wege schleichend ihren Rechtsanspruch auf die Hilfen verlieren, die sie tatsächlich benötigen, und wieder $\mathrm{zu}$ AlmosenempfängerInnen werden. Hierdurch würde die Selbstbestimmung nicht erhöht, sondern der Staat und die Kostenträger würden nur die Verantwortung an den einzelnen behinderten Menschen und an dessen >Community< abgeben. «(Gleiss 2003)

Fatal wäre es, wenn notwendige Hilfen und verlässliche Unterstützungsangebote für Menschen mit Behinderungen im Namen von Community Care und Community Living am Ende immer mehr abgebaut würden - und der Öffentlichkeit dies noch als glänzender Fortschritt in Richtung Teilhabe und Selbstbestimmung verkauft würde. Hier ist endlich auch die Wissenschaft (der Sozialen Arbeit) gefordert, ihre Forschungen, Erkenntnisse und Erfahrungen deutlich zu machen und den Diskurs nicht der Politik und den Sozialverwaltungen zu überlassen.

Die Literaturangaben können beim Autor erfragtwerden (E-Mail jiclausen@web.de).

\section{»eine wertvolle Hilfe»}

Heinz Müller-Dietz, ZS für Strafvollzug und Straffälligenhilfe 2/o4, zur Vorauflage

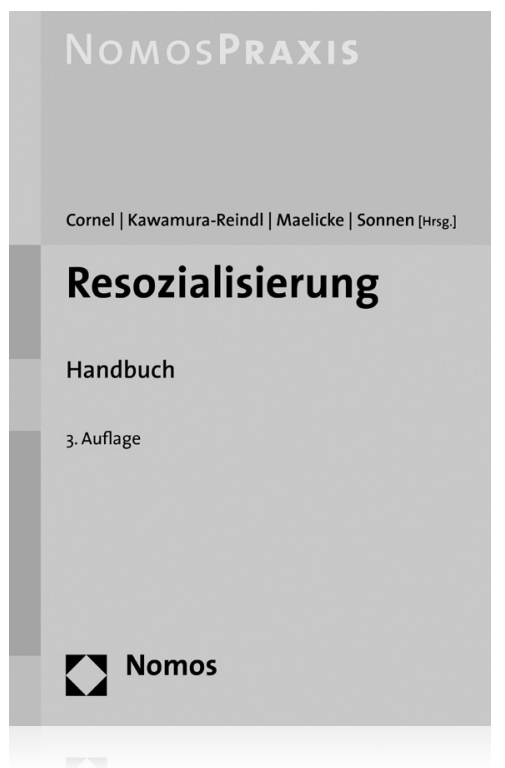

\section{Resozialisierung Handbuch \\ Herausgegeben von Prof. Dr. Heinz Cornel, Prof. Gabriele Kawamura- Reindl, Prof. Dr. Bernd Maelicke und Prof. Dr. Bernd Rüdeger Sonnen 3. Auflage 2008, ca. 600 S., brosch., 59,- $€$, ISBN 978-3-8329-3882-6 Erscheint Dezember 2008}

Die Verwirklichung der Resozialisierung von Straftätern ist in der Praxis des Strafvollzugs tägliche Herausforderung. Die 3. Auflage des Handbuchs vermittelt praxisorientierte interdisziplinäre Fachkenntnisse rund um Erziehung, Sozialisation und Resozialisierung.

\section{Die aktuelle Neuauflage:}

n berücksichtigt die Auswirkungen der Föderalismusreform für den Strafvollzug, Jugendstrafvollzug und Untersuchungshaftvollzug

n stellt dabei die in verschiedene Bundes- und Landesgesetze sowie Verordnungen verstreuten rechtlichen Regelungen der Straffälligenhilfe für Erwachsene, Heranwachsende und Jugendliche übersichtlich dar

- geht auf die Reform der Führungsaufsicht und die Reform des Maßregelrechts ein

- ist um aktuelle Themen erweitert wie z.B. Resozialisierung von Straftätern nicht-deutscher Nationalität, Täter-Opfer-Ausgleich oder Hilfen für Angehörige Inhaftierter

Die Erläuterungen zeigen dabei mögliche Resozialisierungsmaßnahmen und Hilfeleistungen für Straffällige auf.

\section{Nomos}

Bitte bestellen Sie per Fax 07221 / 2104-43, im Buchhandel oder versandkostenfrei unter $\wedge$ www.nomos-shop.de 